Revue d'histoire de l'Amérique française

REVUE D.HISTOIRE DE L'AMÉRIQUE FRANÇAISE

\title{
L'annexion de la côte du Labrador à Terre-Neuve en 1763
}

\section{Gordon O. Rothney}

Volume 17, numéro 2, septembre 1963

URI : https://id.erudit.org/iderudit/302276ar

DOI : https://doi.org/10.7202/302276ar

Aller au sommaire du numéro

Éditeur(s)

Institut d'histoire de l'Amérique française

ISSN

0035-2357 (imprimé)

1492-1383 (numérique)

Découvrir la revue

Citer cet article

Rothney, G. O. (1963). L'annexion de la côte du Labrador à Terre-Neuve en 1763. Revue d'histoire de l'Amérique française, 17(2), 213-243.

https://doi.org/10.7202/302276ar d'utilisation que vous pouvez consulter en ligne.

https://apropos.erudit.org/fr/usagers/politique-dutilisation/ 


\section{L'ANNEXION DE LA COTE DU LABRADOR À TERRE-NEUVE EN 1763}

Deux siècles exactement se sont écoulés depuis que la côte du Labrador a été annexée pour la première fois au gouvernement de Terre-Neuve. Cette décision, prise en 1763, était un résultat de la guerre de Sept Ans. Avant 1759, le Québec avait appartenu à la France vaincue. Terre-Neuve, cependant, avait appartenu depuis 1713 à la Grande-Bretagne victorieuse, et était dirigée en grande partie dans l'intérêt des propriétaires de navires et pêcheurs qui, en fait, vivaient en Angleterre. Les gouverneurs de Terre-Neuve étaient des officiers de marine britanniques et, pendant les mois d'hiver, ils passaient également leur temps en Angleterre. En réalité, on considérait Terre-Neuve dans une large mesure comme une partie de la Grande-Bretagne elle-même. En 1763, les fonctionnaires de Londres étaient très au courant des objectifs que visait le gouvernement britannique à TerreNeuve, mais ils n'avaient pas encore eu le temps d'adapter la politique britannique aux besoins de leur territoire nouvellement conquis du Québec. Par conséquent, il n'est pas du tout étonnant qu'un des résultats de la défaite de la France par la GrandeBretagne en 1763 ait été que le Québec français dut céder une partie de son territoire au territoire britannique de TerreNeuve.

La frontière de 1763 devait subir plusieurs modifications au cours des années qui suivirent. Il reste que, dans l'ensemble, la décision de 1763 a survécu. Aujourd'hui, le Québec et Terre-Neuve sont unis sur un pied d'égalité en tant que provinces du Canada. Les guerres qui, dans le passé, en faisaient des ennemis, sont depuis longtemps révolues. Mais, par suite de la découverte récente de vastes quantités de minerai de fer, la péninsule du Labrador a acquis soudainement le caractère d'une région de grande valeur et de grande importance. Aussi, les 
mêmes circonstances exactement qui, au début, amenèrent sa répartition entre ces deux gouvernements, sont-elles devenues une question d'actualité.

\section{I. - TERRE-NEUVE ET LABRADOR, 1708-1762}

Lorsque Terre-Neuve devint partie intégrante du Canada en 1949, on procéda à une annexion fondée sur les faits géographiques qui existaient déjà en 1708. Cette année-là, le fort anglais de St. John's se rendait à une force française de Plaisance (Placentia). A l'époque, comme aujourd'hui, toute la région de l'Ottawa et du St-Laurent, ainsi que son prolongement occidental au-delà du lac Supérieur, se trouvaient ainsi unis, tant du point de vue politique que du point de vue économique. Ne fut-ce que pour une brève période de temps, l'ensemble de TerreNeuve était devenu partie intégrante du Canada, - le Canada de Louis XIV.

Mais le Canada, à l'époque, pouvait être maîtrisé de la mer. A mesure que la Marine britannique devenait plus puissante que celle de la France, cette vaste région changea graduellement de mains. Le processus commença en Nouvelle-Écosse avec la reddition de Port-Royal en 1710. En 1713, le traité d'Utrecht accentuait cette évolution :

L'île de Terre-Neuve ... appartiendra à la GrandeBretagne... Dans ladite île, il ne sera pas permis auxdits sujets de la France de pêcher et de sécher le poisson en aucune autre partie, que depuis le lieu appelé Cap de Bona Vista, jusqu'à l'extrémité septentrionale de ladite île, et de là, en suivant la partie occidentale, jusqu'au lieu appelé Pointe-Riche.

Le Labrador, toutefois, demeurait sous le contrôle du Québec et de la France. Des postes de traite de fourrures avaient été établis le long de la rive nord du golfe St-Laurent, de même que le long de la côte atlantique septentrionale jusqu'à la baie des Esquimaux. Ces postes étaient devenus suffisamment importants pour réclamer la surveillance de la part d'un représentant spécial du Roi. Le 12 novembre 1714, le décret suivant était promulgué à Marly "de par le Roi": 
Sa Majeste estimant necessaire qu'il y ait un officier de ses troupes pour commander a la coste de Labrador pays des equimaux et estant satisfaite des fermiers du Sieur de Courtemanche Capitaine d'une de ses compagnies entretenues en la nouvelle france qui a deja fait un etablissement a la dite coste, Sa Majesté veut et entend qu'il commande en la dite coste de Labrador et qu'il y regle et accomode les differents qui pourront arriver entre les sujets de Sa Majeste au sujet des emplacements pour la pesche a la dite coste mande Sa Majesté au Sieur Marqui de Vaudreuil gouverneur et lieutenant general en nouvelle france de le faire reconnoitre en la dite qualité et a tous autres qu'il appartiendra de luy obeir et entendre ${ }^{1}$.

Le même jour, le Roi

estant informé de la reussite de l'etablissement que le Sieur de Courtemanche a fait a la baye de Phelypeaux coste de Labrador pays des esquimaux ou il luy a esté accordé en l'année 1702 une concession pour l'espace de dix années par les sieurs de Vaudreuil et Beauharnois gouverneur et intendant en la nouvelle france... avec pouvoir au dit Sieur de Courtemanche seul d'y faire la traitte avec les sauvages, la pesche de Baleines, loups marins et Morues, Sa Majesté... luy a concedé par le present brevet la baye de Phelypeaux ou il est presentement etabli avec...2 lieues en remontant du costé de Quebec et deux lieues en descendant du costé du detroit de Belisle ... pour en jouir sa vie durant..., veut Sa Majesté qu'il ait seul... la faculté d'y faire la pesche du loup marin, et qu'a l'egard des autres pesches qu'il les fasse concurramment avec les vaisseaux qui viendront a la dite Baye.

Comme quartier général, Courtemanche choisit le petit fort Pontchartrain dans la baie de Phelypeaux (Bradore Bay), qui se trouve un peu du côté québecois de la frontière actuelle de Terre-Neuve au Labrador. A sa mort en 1717, son beau-fils, le Sieur de Brouage, était nommé pour lui succéder, et il conserva

${ }^{1}$ In the Privy Council. In the Matter of the Boundary Between the Dominion of Canada and the Colony of Newfoundland in the Labrador Peninsula ..., 1927, Volume VII of Joint Appendix: 3574 . Ci-après Joint App. 
ce commandement jusqu'au début de la guerre de Sept Ans en $1756^{2}$.

Entre-temps, on savait très peu de choses du Labrador en Grande-Bretagne, bien qu'en 1747 un géographe de Londres eût parlé de "ce pays que les Anglais appellent Nouvelle-Bretagne "". En 1752, plusieurs marchands londoniens soumirent une pétition au gouvernement en vue de se faire concéder toute la côte atlantique du Labrador afin d'y établir des colons et d'ouvrir un nouveau commerce de fourrures, de nageoires de baleines, de poissons et de mâts de navires, - et de persuader les Esquimaux de combattre contre les "Indiens français" (Montagnais) ${ }^{4}$. A Whitehall et à Westminster, lord Halifax, Charles Townshend, et les autres membres du Conseil consultatif du commerce et des plantations examinèrent "tout ce qui figurait dans les livres de notre bureau" à propos de la "Nova Brittannia ou Terra Labrador" et rapportèrent que les géographes français séparaient cette région des établissements français le long du StLaurent par une ligne partant du détroit de Belisle et se dirigeant vers l'ouest à travers le continent. "Il ne semble pas, déclarèrentils, qu'aucun établissement permanent n'ait jusqu'ici été fait par aucune nation dans les régions orientales de ce pays", les Anglais "qui furent les premiers navigateurs dans cette partie du monde" s'étant dirigés vers la baie d'Hudson, alors que les Français, à cause de "l'hostilité implacable des Indigènes, n'ont pas encore installé d'établissements au nord du Détroit de Belle Isle". Ce manque complet de renseignements sur l'activité française au Labrador révèle qu'au milieu du dix-huitième siècle le gouvernement britannique n'avait aucun contact avec ce littoral.

En ce qui concerne l'histoire primitive du Labrador, les connaissances $\mathrm{du}$ Conseil $\mathrm{du}$ commerce étaient également inexactes :

${ }^{2}$ Archives du Canada, série C 11 6, VIII : 151; Joint App. VII : 3575-6. Sam. Edw. Dawson, "Brest on the Quebec Labrador", Société Royale du Canada, Proceedings and Transactions, 1905, partie II, page 26.

3 Emanuel Bouman, A Complete System of Geography, II: 767 (Londres, 1747), (Joint App., VIII : 3782).

4 Journal of the Commissioners for Trade and Plantations, Jan.1749/50 to Dec. 1753, 299-300, 16 April, 1752. 
Le premier récit authentique que nous ayons d'une découverte de ce pays est celui de Sébastien Cabot, en date de 1497, qui avait reçu d'Henri VII la mission de faire des découvertes vers l'Ouest. $\mathrm{Au}$ cours du premier voyage qu'il entreprit, aux termes de cette mission, il entra en contact avec le territoire situé au $67^{\circ}$ de latitude nord, d'où il poursuivit sa route vers le sud le long de la côte du Labrador jusqu'à Terre-Neuve...

En réalité, 1497 est l'année non pas du voyage de Sébastien, mais de son père, Jean Cabot. Cette erreur avait déjà figuré dans une déclaration faite par le $\mathrm{D}^{\mathrm{r}}$ John Dee, dès 1578, qui voulait ainsi prouver que la Reine Élisabeth $\mathrm{I}^{\text {re }}$ avait un titre à l'Amérique du Nord par droit de découverte. Ce prétendu titre était encore considéré comme "authentique" par les fonctionnaires britanniques en 1752. La véritable date du voyage de Sébastien Cabot dans la baie d'Hudson est $1509^{5}$. Les connaissances $d u$ Conseil du commerce, tant en ce qui concerne l'histoire du Labrador que de son état véritable à l'époque, étaient négligeables. Néanmoins, la conclusion qu'il avait tirée en 1752 révèle que le gouvernement britannique croyait que la situation au Labrador, immédiatement avant la conquête du Québec, était la suivante:

que le titre aux régions orientales de Terra Labrador où les pétitionnaires se proposent d'installer un établissement, n'avait jamais fait l'objet d'aucun différend ou d'aucune discussion, avec tout autre prince ou Etat, les différends intervenus avec la couronne de France dans les années 1687, 1699 et 1719 ayant été uniquement limités à la baie d'Hudson et au territoire qui en dépend, mais il semble toutefois que bon nombre des arguments invoqués et des preuves présentées de part et d'autre à l'occasion de ces différends, impliquent effectivement, si l'on tient compte de leur nature, des prétentions beaucoup plus étendues, et tendent à influer sur le titre à l'ensemble de cette partie de l'Amérique du Nord, et il ne semble pas que ces différends aient à aucun moment été réglés de façon définitive ou

5 James A. Williamson, The Cabot Voyages and Bristol Discovery under Henry VII (Cambridge, 1962), 28, 170, 201-202. 
qu'une ligne de frontière ait été fixée entre les Dominions de Sa Majesté et ceux de Sa Majesté très chrétienne dans ces territoires.

Pour ce qui est de la Compagnie de la Baie d'Hudson, tout ce qui la préoccupait c'était d'empêcher d'autres marchands londoniens de concurrencer son propre commerce dans la péninsule du Labrador. Par conséquent, déclarait le Conseil du commerce, si toute concession devait être accordée sur la côte du Labrador, il serait nécessaire "que pareille concession fasse l'objet d'une limite occidentale, n'empiétant pas sur la charte et la propriété de cette compagnie ${ }^{6}$ ". C'est le fait de n'avoir pu, en 1763, ou plus tard, préciser cette limite occidentale qui rendit nécessaire la décision du Conseil privé en 1927.

Les Français, d'autre part, considéraient tout ce territoire comme faisant partie du Canada. En 1754, le sieur Boucault écrivait notamment: "Le Canada, ou la Nouvelle-France" comprend "la terre ferme de Labrador, et toutes les côtes maritimes qui se terminent et s'étendent de Détroit de Belle-Isle jusqu'à la Baye d'Hudson "'. Et, en 1755, certaines Remarques sur la Carte de l'Amérique Septentrionale étaient publiées à Paris par M. Bellin, "Ingénieur de la Marine \& du Dépôt des Cartes, Plans \& Journaux, Censeur Royal, de l'Académie de Marine, \& de la Société Royale de Londre". A propos du "Voyage que Jean Cabot, Vénitien, entreprit en 1497", il dit:

Ce Navigateur vit en passant les Côtes Orientales de l'Isle de Terre Neuve et quelques parties du Continent voisin, mais il ne débarqua en aucun endroit de l'Ile ni du Continent, et les Anglais ne peuvent rien montrer qui puisse le prouver; ...

Bellin savait du moins que c'était Jean et non pas Sébastien qui avait entrepris ce voyage de 1497, bien qu'il fût dans l'erreur lorsqu'il déclarait qu'il n'y avait pas eu de débarquement.

6 Public Record Office, Londres, C.O. 5/6. Lords du Commerce aux Lords Juges, 23 juillet 1752 (Joint App., III: 883-892). Ci-après, tous les documents mentionnés se trouvent au Public Record Office, Londres, à moins d'indication contraire.

7 Rapport de l'Archiviste de la Province de Québec, 1920-1921, 11, "Etat présent du Canada" (Joint App., VIII: 3780). 
Cependant, en 1755, il était vrai que le gouvernement britannique n'avait aucune preuve de ce que fit Jean Cabot en 1497 de ce côté-ci de l'océan. Le Canada ou la Nouvelle-France, disait Bellin, comprenait Terre-Neuve (cédée aux Britanniques en 1713 "avec réserve") et le Labrador:

$\mathrm{Au}$ Nord du Détroit de Belle-Isle sont les Côtes de Labrador, grand \& vaste Pays, que les François avoient nommé anciennement nouvelle Bretagne; mais le nom de Labrador ... a prévalu. Les François ont seuls droit sur ces Pays, puisqu'ils l'ont découvert en meme tems que le Golfe Saint Laurent dont il fait partie, \& que dés les premiers tems ils ont commercé \& qu'ils y commercent encore seuls avec les Naturels du pays ...8

La même année, soit en 1755, J. Palairet, agent hollandais, publiait à Londres $A$ Concise Description of the English and French Possession in North America. "La Nouvelle-Bretagne", écrivait-il, a été divisée en deux parties par la baie d'Hudson: "Ce territoire a été appelé Nouvelle-Bretagne par les Anglais. La partie de ce territoire qui se trouve à l'est de la Baie d'Hudson a été appelée Labrador par les Espagnols "'. C'était la période de la "guerre froide" qui précéda l'ouverture officielle des hostilités en 1756, mais bien que les Français et les Britanniques fissent part de leurs prétentions au titre de la côte du Labrador, ni les uns ni les autres ne prétendaient que ce territoire dût être rattaché à Terre-Neuve, ou qu'il l'eût jamais été.

Après la capitulation de Plaisance, capitale de Terre-Neuve en 1713, les Français avaient commencé à construire une forteresse beaucoup plus puissante à Louisbourg, dans l'île du CapBreton, dans l'espoir que, de là, ils pourraient empêcher la conquête britannique du Canada de s'étendre plus loin. Ils auraient été plus sages d'investir cet argent dans leur Marine. Car les soldats de Louisbourg ne pouvaient empêcher les Britanniques d'entreprendre la construction d'une forteresse à Halifax en 1749 pour défendre leurs possessions contre les

8 Joint App., VIII : 3774-3777.

9 Joint App., VIII: 3783. 
Français. Malheureusement, les "moyens de dissuasion" ne dissuadent jamais. Ils ne font qu'accroître la tension. Les Acadiens devinrent nerveusement hostiles et furent "expulsés" de la Nouvelle-Écosse et puis, en 1758, une expédition d'Halifax captura Louisbourg. Les Britanniques avaient alors entrepris de parachever la conquête du Canada, commencée en 1710.

En 1759, deux navires de guerre furent envoyés sur la côte du Labrador pour y dépister tout Français qui aurait pu encore s'y trouver. Des maisons et des magasins de la rive nord du golfe furent brûlés ou détruits tout le long de la côte jusqu'au détroit de Belle Isle ${ }^{10}$. En 1760, à Montréal, la conquête était parachevée.

Tout le Canada, de Terre-Neuve jusqu'à l'ouest des GrandsLacs, se trouvait de nouveau uni sous un seul souverain, comme il l'avait été sous Louis XIV en 1708. Versailles avait été remplacée par Westminster en tant que capitale de tout le pays, tout comme, sur le plan local, Plaisance avait été remplacée par St. John's en tant que capitale de Terre-Neuve et Port-Royal par Halifax en tant que capitale de la Nouvelle-Écosse. Mais ce que les Britanniques ne firent pas, comme les Français l'avaient fait avant 1710, ce fut d'instituer une autorité suprême au Canada même. Ce n'est qu'en 1949 qu'Ottawa parvint enfin à occuper la situation qu'avait occupée le Québec en tant que capitale centrale de tout le territoire de ce qui avait déjà été la Nouvelle-France. Les Britanniques s'étant emparés du Canada par petits morceaux, à différentes périodes, et comptant déjà bon nombre de gouvernements coloniaux complètement distincts en Amérique du Nord, n'avaient aucune conception de l'ensemble en tant qu'unité compacte, jusqu'à ce qu'ils fussent forcés de s'en rendre compte par suite des résultats stratégiques de la Révolution américaine. La logique de la géographie et de l'économie s'affirma de nouveau enfin par le moyen de la Confédération. Mais, dans l'entre-temps, il n'y avait pas eu d'autre autorité plus rapprochée que celle de Londres pour régler les différends qui, inévitablement, éclataient entre les colonies distinctes de

10 Ad. 1/482, 127-130, Saunders à Cleveland, 24 novembre 1759. 
l'Amérique du Nord. L'un de ces différends intéressait le Québec et Terre-Neuve dans la péninsule du Labrador.

Après la capitulation de Québec, on présumait dans cette ville que le Labrador relevait de l'autorité du gouverneur militaire, James Murray:

Peu après la capitulation du Canada, plusieurs commerçants se rendirent à Québec, de Grande-Bretagne, avec de grandes quantités de marchandises. On constata bientôt que les produits du Canada et de ses dépendances étaient beaucoup moins considérables qu'on ne l'avait d'abord imaginé. Anciennement, le commerce avec les sauvages des pays d'en haut, de Montréal jusqu'au fort Illinois, s'était poursuivi avec succès, et s'était accru en grande partie grâce aux Canadiens, mais depuis la capitulation, les interruptions causées par la guerre indienne, et la participation des autres colonies britanniques à ce commerce réduisirent considérablement sa valeur pour le Canada, de sorte que les nouveaux colons, ayant de la difficulté à faire passer leurs produits en Grande-Bretagne, et voyant que la pêche aux phoques était abandonnée par suite de la guerre, qui avait empêché les Canadiens de la poursuivre, demandèrent au gouverneur de Québec la permission de s'engager dans ce commerce ${ }^{11}$.

Murray accéda aussitôt à la demande des marchands de langue anglaise nouvellement arrivés. Il croyait que le commerce du phoque offrait de grandes possibilités. Il désirait également trouver de l'emploi pour les Canadiens français qui étaient devenus experts à la pêche au phoque avant la guerre, mais qui étaient désormais sans travail par suite du manque de capitaux. C'est ainsi que, pour la première fois, des marchands de langue anglaise ("anciens sujets") devinrent propriétaires de terres sur la côte du Labrador, à côté des propriétaires de langue française ("nouveaux sujets"), dont les possessions avaient été garanties par les termes de la capitulation de Montréal en 1760.

11 C.0. 194/18, 272. "Case of the Landholders in Canada..." (Pièce jointe à une "Note from the Merchants interested in the Trade to Quebec", 19 mars 1768). Imprimé dans Joint App., III: 1025-1026, de "B.T. Newfoundland, Vol. 20". 
L'un des premiers à bénéficier de cette politique fut Joseph Isbister, qui put ainsi louer le poste de Mingan, sur la côte nord du golfe, des propriétaires de la seigneurie de TerreFerme-de-Mingan. Voici ce que déclarait le général Murray à ce propos:

Que j'aie effectivement recommandé M. Isbister à M. Lafontaine et à M. Tachet, comme étant l'homme le mieux indiqué pour le poste de Mingan, il n'est rien de plus certain; ce M. Isbister avait longtemps été principal gouverneur de la baie d'Hudson, il est arrivé ici en 1760 avec sa femme et six jeunes enfants, avait des marchandises au montant de 900 livres, lesquelles étaient entièrement calculées en vue des postes situés plus bas. Comme il ne m'avait pas été recommandé, c'est par compassion pour lui que je voulus mettre cet homme en mesure de gagner sa vie. C'était pour moi de bonne politique que de vouloir l'établir dans les postes plus bas, vu qu'il avait le caractère d'un honnête homme et qu'il connaissait parfaitement la langue des Indiens habitant là, et la prudence exigeait en ce temps de guerre qu'on sût bien quels étaient ceux qui occupaient les postes dans le golfe et le fleuve St-Laurent, de sorte que des passeports étaient nécessaires ${ }^{12}$.

Comme la demande était grande en Europe pour l'huile et les fanons de baleine, Murray désirait encourager les pêcheurs de baleine. Le 21 août 1761, il concédait pour une période de trois ans une étendue de terre sur la rive nord du golfe St-Laurent à la Whale Fishing Company ${ }^{13}$, formée de marchands de langue anglaise de Québec. Mais la guerre n'était pas encore terminée, même sur la côte du Labrador. Lafontaine, l'un des nombreux propriétaires conjoints de la seigneurie de Mingan, ayant conclu son marché avec Isbister en 1761, se rendit cet automne-là à un autre de ses postes sur la côte, où sa conduite incita le général Murray à conclure qu'il était "un ennemi de l'État":

... d'après les renseignements que nous avons eus cet hiver concernant des corsaires et des pirates qui

${ }^{12}$ Archives du Canada, Série Q, Vol. 2. Réponse publique de James Murray, 24 avril 1764 (Joint App., VII: 3549).

13 Joint App., VII : 3564. Rapport sur les postes ou terres concédés par le gouverneur Murray ... jusqu'au 22 juillet 1763. 
s'abritaient et gagnaient leur vie au moyen des postes et de la Baie des Chaleurs, j'en vins à croire qu'il était hautement souhaitable, à la demande de tous les marchands d'ici, d'armer un navire en vue de la protection de leur commerce et de la surveillance des postes, de même que de redoubler de précaution à l'égard de ceux qui devaient occuper les postes situés dans le golfe et le fleuve St-Laurent. Par conséquent, il devint opportun d'enlever le poste de Mekatinat de La Fontaine pour le donner à $M$. Gray, en qui je pouvais avoir confiance ${ }^{14}$.

En 1761, William Pitt, à qui était attribuable la conquête du Canada, fut remplacé comme secrétaire d'Etat par lord Egremont. Le 5 juin 1762, Murray, en sa qualité d'un des trois gouverneurs militaires, soumettait à la demande d'Egremont un "compte rendu du gouvernement de Sa Majesté à Québec et dans ses dépendances", qui devait inciter le Conseil du commerce à formuler une recommandation à l'égard du Labrador, dont le général n'avait jamais rêvé. Parlant des nations indiennes habitant dans le territoire qui relevait du gouvernement, il signalait d'abord les "sauvages de la rive nord", (les Esquimaux): "Durant l'été, ils viennent avec toutes leurs familles en chaloupe pour pêcher dans les détroits de Belisle; ils y laissent leurs familles dans les baies, et se rendent euxmêmes, à une distance considérable en canot." Ils "avaient rencontré à maintes reprises les Français et les Canadiens qui s'adonnaient à la pêche dans ces régions... Quelques-uns ici ont trafiqué avec eux et y ont retiré de gros avantages...".

Deuxièmement, poursuivait Murray, les Montagnais "habitent une vaste étendue de territoire, du Labrador jusqu'au Saguenay". Ceux "qui vivent à l'intérieur des terres... commercent souvent" du côté de la baie d'Hudson, ce que "les habitants du côté de la mer... auraient été obligés de faire, si l'interruption causée par la guerre s'était poursuivie le moindrement longtemps, quoique, la situation étant plus commode, ils se seraient constamment adressés à ceux qui étaient maîtres du fleuve St-Laurent ...”.

${ }^{14}$ Archives du Canada, Série Q, Vol. 2, Réponse publique de James Murray, 1764 (Joint App., VII: 3550). 
Sous la rubrique intitulée "Commerce", Murray déclarait ce qui suit:

On pourrait établir un commerce de pêche à la morue des plus considérables et des plus étendues dans le fleuve et le golfe du St-Laurent... le poisson pêché sur ces côtes et dans les baies excède de beaucoup en quantité la morue pêchée sur les bancs, et commande un prix avantageux sur les marchés étrangers; les pêcheurs étant sur place commenceront à pêcher dès que la saison le permettra et continueront à le faire jusqu'à la fin de la saison, gagnant ainsi au moins deux mois dans ce commerce...

Bien que Murray songeât principalement à la possibilité d'installer des établissements du côté sud du golfe, ces observations n'étaient pas de nature à soulever beaucoup d'enthousiasme parmi les hommes d'État britanniques qui estimaient que la force de la Marine britannique dépendait de la prospérité des pêcheurs résidant en Angleterre pendant l'hiver, et traversant l'océan pendant l'été seulement.

Dans sa conclusion, Murray faisait observer, en ce qui concerne les limites géographiques de son gouvernement,

à quel point il est impossible de vérifier exactement quelle partie de l'Amérique du Nord les Français ont appelée Canada, aucune carte n'étant tombée entre nos mains ni aucunes archives ne pouvant montrer ce qu'ils entendaient par là.

Cependant, il est à espérer que les limites de ce côté-ci du moins ne nécessiteront aucune vérification ni ne donneront lieu à aucun différend ${ }^{15}$.

Murray devait être déçu dans son espoir. A sa grande surprise, les limites de la partie orientale du Canada furent effectivement non seulement vérifiées, mais tracées de telle façon qu'elles devaient donner lieu à un différend avec le gouverneur de Terre-Neuve.

15 C.O. 323/15, Q. 31, Murray à Egremont, 5 juin 1762. (Joint App., III : 893-898, de "Documents constitutionnels (1759-1791), $2^{\mathrm{e}}$ édition, page 47, Archives du Canada"). 
Dans l'intervalle, la pêche du phoque au Labrador, du détroit de Belle Isle jusqu'au Cap St-Charles sur la côte atlantique, avait été bientôt réorganisée par les marchands de langue anglaise de Québec. Bien qu'ils eussent travaillé à crédit, et que les recettes des quelques premières années fussent absorbées par les frais initiaux, tout semblait indiquer que les marchands retireraient bientôt des bénéfices suffisants pour leur permettre de rembourser leurs dettes en Grande-Bretagne.

\section{II. - LA DÉCISION DE 1762-1763}

Les préliminaires de paix entre la Grande-Bretagne et la France furent conclus le 3 novembre 1762, et le traité définitif fut signé à Paris le 10 février 1763, ce qui mettait fin à la guerre de Sept Ans. La Grande-Bretagne était sortie non seulement victorieuse de cette guerre, mais incontestablement la plus grande puissance du monde. Mais, à la fin de la guerre, elle se trouvait indécise quant à la façon d'administrer ses territoires nouvellement conquis. Par conséquent, le gouvernement britannique soumit la question à son "Conseil du commerce". A l'époque, on considérait l'Empire britannique tout simplement comme un moyen d'accroître le commerce des marchands vivant en Grande-Bretagne.

Il était considéré comme admis à Londres que l'Empire brtannique et, par suite, le commerce britannique, dépendaient de la maîtrise des mers par la Grande-Bretagne. La puissance maritime, d'autre part, dépendait d'un flot continu de marins expérimentés de la Marine britannique, - et l'on avait présumé depuis longtemps que les pêcheries étaient de beaucoup la meilleure "pépinière de marins". On estimait donc que l'exploitation des pêcheries par des pêcheurs vivant en Grande-Bretagne était essentielle à la survivance du commerce britannique et à l'Empire britannique. Par conséquent, l'encouragement aux pêcheries outre-mer, administrées à partir des Îles britanniques, bénéficiait de la plus haute priorité parmi les politiques du gouvernement britannique. Tout le reste, même le principe mercantiliste d'un Empire autarcique, venait en second lieu, lorsque le bien-être des pêcheries de la mère patrie 
était en cause. Les victoires retentissantes remportées par la Marine britannique pendant la guerre de Sept Ans, à compter de la réduction de Louisbourg en 1758 jusqu'à la reprise de St-Jean à Terre-Neuve et à la capture de Cuba en 1762, et la maîtrise absolue de la route des Indes, tout cela semblait justifier l'appui traditionnel accordé aux pêcheries britanniques. On n'était nullement enclin, en 1763, à modifier une ligne de conduite qu'on estimait s'être révélée un succès aussi retentissant.

Dans le passé, les principales victimes de cette attitude avaient été les habitants anglais de Terre-Neuve. Leur île n'avait jamais été considérée comme une "plantation", comme la Nouvelle-Écosse ou le Massachusetts; bien qu'il eût été jugé nécessaire de nommer un gouverneur d'été et certains "juges d'hiver", il n'y avait pas d'assemblée législative, ni même de conseil nommé. Terre-Neuve était considérée comme un territoire de "pêcheries" et non une colonie. N'étant nullement soumises au contrôle du gouvernement, les conditions sociales pendant les mois d'hiver n'auraient pu être pires. Mais, bien que le commerce des pêcheries britanniques eût décliné de façon sérieuse pendant la guerre, il n'y avait aucun doute que les intérêts de ce commerce auraient la priorité sur tout intérêt colonial. Ce principe s'appliquerait au Québec aussi bien qu'aux possessions britanniques plus anciennes.

Même en concluant le traité de paix de 1763, on avait manifesté le même manque habituel de considération pour les intérêts coloniaux à Terre-Neuve. Sous la direction vigoureuse de William Pitt, les victoires britanniques de 1760 avaient porté le prestige de la Grande-Bretagne au point le plus élevé jamais atteint dans toute son histoire. Mais, cette même année-là, c'est-à-dire l'année où le gouverneur de la Nouvelle-France avait capitulé à Montréal, la mort de George II avait fait accéder au trône britannique son incompétent petit-fils, George III. Cet événement se révéla une catastrophe nationale. Le favori du nouveau roi, lord Bute, devint cette année-là, à toutes fins pratiques, premier ministre, bien qu'il ne fût ni membre du Parlement ni du cabinet. En 1761, il fut nommé secrétaire d'Etat. Sa politique était celle de la "paix à tout prix" afin 
que le roi pût appliquer toute son ambition à devenir chef suprême du Parlement. Il ne comprenait nullement les objectifs commerciaux pour lesquels les hommes d'affaires britanniques, dirigés par Pitt, avaient fait la guerre. Pitt n'avait nullement l'intention de songer à la paix tant que la concurrence française dans le monde entier ne serait pas complètement éliminée. Aussi, en octobre 1761, força-t-on Pitt à quitter le gouvernement. N'eût été ce changement, les Français n'auraient pas été autorisés à retourner aux pêcheries de Terre-Neuve. En mai 1762, Bute, en sa qualité de Premier Lord du Trésor, devint premier ministre de nom, de même que de fait.

Quand William Pitt avait démissionné du gouvernement en 1761, il avait été remplacé par le comte d'Egremont comme secrétaire d'État aux Affaires méridionales (chargé des relations avec les nations européennes "méridionales" et du commerce et des colonies d'outre-mer). Bien qu'Egremont ne se fût jamais tout à fait identifié avec les "amis du roi", et bien qu'il ne fût pas tout à fait en faveur de la paix à tout prix, comme Bute, il n'en continua pas moins à détenir son poste important dans le cabinet, lorsque Bute devint premier ministre en 1762, de sorte qu'il partagea la responsabilité des actes de ce gouvernement ${ }^{16}$. En fait, encore plus que Bute, il fut responsable des conditions de paix conclues avec la France, traité obtenu à force de patience par des méthodes qui effaçaient jusqu'à un certain point le prestige acquis par la Grande-Bretagne pendant la guerre de Sept Ans. C'est ce traité qui accorda St-Pierre-etMiquelon à la France, en compensation de la perte de l'île du Cap-Breton, consommée dès 1758. Mais ce qui était plus grave encore, ne tenant nullement compte du fait que, pendant la guerre, des pêcheurs de langue anglaise avaient occupé presque tous les ports de Terre-Neuve, du cap Bonavista jusqu'au cap St-Jean, ainsi que de nombreux ports septentrionaux, du cap St-Jean jusqu'à Quirpon, le traité de Paris stipulait ce qui suit:

Les sujets de la France auront la liberté de la pêche et de sécherie, sur une partie des côtes de l'île de

16 Dictionary of National Biography, articles sur John Stuart, troisième comte de Bute, et sir Charles Wyndham, deuxième comte d'Egremont. 
Terre-Neuve, telle qu'elle est spécifiée par l'article XIII du traité d'Utrecht ${ }^{17}$;

c'est-à-dire du cap Bonavista jusqu'à l'extrémité septentrionale de l'île, et de là vers l'ouest jusqu'à Pointe Riche. De cette façon, avec d'autres intérêts, ceux des sujets britanniques de Terre-Neuve furent sacrifiés par Bute et Egremont afin d'obtenir une paix rapide avec le roi de France, pour George III. Ce fut le genre d'apaisement, qui, en définitive, signifie non pas la paix mais de nouvelles frictions et la reprise de la guerre. Étant donné l'échec complet de la descente des Français sur St-Jean plus tôt en 1762, de telles concessions dans les pêcheries de Terre-Neuve n'étaient nullement nécessaires. Ce ne fut qu'en recourant à la subornation et à la corruption la plus éhontée que Bute et Egremont purent amener le Parlement à approuver leurs conditions de paix.

Le traité définitif fut signé le 10 février 1763 et, le 8 mars, lord Egremont adressait des copies des articles relatifs aux pêcheries aux lords Commissaires du commerce et des plantations, leur enjoignant de

soumettre au roi en son conseil toute modification ou addition qui pourrait sembler à Vos Excellences opportune dans les instructions données au gouverneur de Terre-Neuve afin de les conformer aux stipulations ci-dessus du traité définitif ${ }^{18}$.

Il se trouva qu'un nouveau président du Conseil du commerce avait été nommé à la fin de février 1763 , et ce fut là l'un des premiers problèmes qu'il eut à résoudre. Il s'agissait de Charles Towshend, alors jeune politicien d'environ trente-huit ans. De façon générale, il était partisan de William Pitt, bien qu'à certains moments il semblât plutôt vacillant et sans caractère. Néanmoins, il avait été nommé membre du Conseil privé en 1757 , et avait détenu une fonction sans importance pendant le long gouvernement de Pitt (1757-61). Il avait été promu secrétaire d'État à la Guerre en 1761 , mais avait démissionné peu après que Bute fut devenu premier ministre en 1762, pour se joindre

17 G. F. de Martens, Recueil de Traités (2édition), I: 109 (article 5). 18 C.0. 194/15 f. 59, Egremont aux Lords du Commerce, 8 mars 1763. 
à l'Opposition avec Pitt. Par conséquent, il n'était nullement responsable des conditions de paix. Cependant, une fois signé le traité définitif mettant fin à la guerre, il était disposé à occuper de nouveau une fonction, de sorte qu'il fut presque aussitôt nommé président du Conseil du commerce et des plantations. En réalité, il n'occupa cette fonction que pendant un peu plus d'un mois avant de rejoindre Pitt dans l'Opposition. Mais ce fut au cours de ce mois que fut prise l'importante décision de rattacher le Labrador à Terre-Neuve ${ }^{19}$.

L'appui accordé à Pitt, et son opposition en 1762 au Roi, à Bute et à Egremont, révèlent que Charles Townshend n'était pas partisan d'un "apaisement" avec la France. D'autre part, il lui manquait la largesse d'esprit et le libéralisme de Pitt envers les habitants des colonies d'outre-mer. On devait en avoir une preuve concluante au moment du dernier geste important de sa vie, lorsqu'il fut de nouveau en fonction, en qualité de chancelier de l'Échiquier sous le régime de Pitt en 1767. Pendant la maladie du premier ministre, ce fut lui qui eut à saisir le timon des affaires et imposa une taxation indirecte sur le thé et certains autres articles dans les colonies, bien que son propre chef fût l'adversaire anglais le plus résolu de pareille politique. Townshend était destiné à mourir soudainement, sans savoir qu'il avait provoqué chez les coloniaux une fureur qui ne devait s'éteindre qu'avec la victoire de la Révolution américaine. Il est instructif de noter, à l'égard des aptitudes des trois hommes les plus directement responsables de la politique d'outre-mer en mars 1763, que, à eux trois, ils représentaient toutes les attitudes d'esprit qui, en définitive, aboutirent à la dislocation de l'Empire britannique, c'est-à-dire Bute, Egremont et Townshend.

$\mathrm{Au}$ lieu de soumettre au Cabinet des instructions supplémentaires qu'il fallait donner, estimaient-ils, au gouverneur de Terre-Neuve, comme le demandait lord Egremont, Townshend et ses collègues du Conseil du commerce formulaient le 15 mars 1763 certaines opinions à l'égard de la nécessité de telles instruc-

19 Dictionary of National Biography, article sur Charles Townshend (1725-1767). 
tions. Leurs observations ne renfermaient aucune référence aux intérêts des habitants de Terre-Neuve ou de toute colonie. Conformément à la coutume traditionnelle, le mot "Terre-Neuve" ne signifiait pour eux qu'un territoire de pêche, c'est-à-dire tout le territoire de pêche de Terre-Neuve, qu'il dépendît directement de l'île ou non. C'est cette conception de Terre-Neuve en tant que "territoire de pêche" qui rendit si facile l'extension de l'autorité du gouverneur aux régions adjacentes, — régions qu'on pouvait atteindre à partir de l'île principale, par eau, et non par terre.

Dans leurs doléances du 15 mars 1763, les Lords du Commerce formulèrent deux observations principales concernant les effets du traité de Paris. La première était que

le territoire général des pêcheries de Terre-Neuve, et le golfe et les côtes qui l'entourent sont devenus une question d'une portée infinie, et de la plus haute importance;

la guerre ayant ouvert "la partie nord-est de Terre-Neuve", ainsi que le "vaste territoire étendu de la pêche à la baleine dans le détroit de Belle Isle, y compris les autres territoires de la pêche au saumon et au phoque, qui s'étend sur la côte à partir de l'embouchure de ce détroit jusqu'au fleuve St-Laurent", et aussi "le territoire de pêche du fleuve St-Laurent lui-même, de Gaspé, de Canceaux, des Iles-de-la-Madeleine, de St-Jean et du Cap-Breton".

La seconde observation formulée par Charles Townshend et ses collègues du Conseil du Commerce concernait les Français: empêcher les Français de partager les avantages de ces pêcheries... nous semble rendre absolument nécessaire l'adoption de quelques mesures additionnelles et instructions supplémentaires, en vue de l'exécution du traité définitif ... afin d'assurer la possession exclusive de ces pêcheries précieuses aux sujets de Votre Majesté.

C'est à cet égard que la seule mention précise du Labrador fut faite. Mais cette seule mention amena l'annexion de cette côte à Terre-Neuve, et révèle la raison pour laquelle la mesure initiale fut prise : 
Sur la côte du Labrador, il sera impossible d'empêcher les Français de continuer à bénéficier pleinement des avantages de leur ancien commerce avec les Indiens de cette côte, à moins qu'un établissement britannique quelconque s'y installe, ou qu'un nombre suffisant de croiseurs soient stationnés avec instruction à leur commandant de saisir tous navires français approchant d'une certaine distance de cette côte.

Le Conseil ne suggérait pas, dans ce document, l'annexion du Labrador à Terre-Neuve, mais déclarait effectivement qu'on ne pouvait empêcher le commerce français dans ce territoire que de l'une des deux façons suivantes: soit au moyen d'un "établissement britannique", soit au moyen d'un nombre "suffisant de croiseurs".

A ce stade-là, Charles Townshend et ses collègues se contentèrent de soumettre ces observations au Roi en son conseil, ajoutant que les mesures qu'ils recommandaient devaient "faire partie d'un plan général", qui dépendrait "des efforts concertés", de "différents ministères du gouvernement 20". Les Lords du Commerce affirmaient, en effet, que par suite du traité de paix, le territoire général de pêcherie à la disposition des sujets britanniques était devenu "infini" et qu'ils ne pouvaient donner de nouvelles instructions au gouverneur de Terre-Neuve tant que la politique de base à cet égard n'aurait pas été décidée dans son ensemble par le gouvernement.

Cette réponse n'impressionna guère lord Egremont. Il était trop pressé pour attendre la mise en place d'un "plan général", tout sage que pût être cette initiative. Au lieu de cela, il répondit au Conseil du commerce, le 17 mars 1763, en ces termes:

Sa Majesté ayant pris en considération vos doléances du 15 courant ne juge pas opportun que des mesures soient prises à cet égard, jusqu'à ce que Vos Excellences se soient pleinement conformées aux directives découlant des Ordres de sa Majesté, et contenues dans ma lettre du $8 \ldots$ c'est l'intention du Roi

20 C.O. 194/26, f. 69. Lords du Commerce à Egremont, 15 mars 1763. (Joint App., II : $386-8$ ). 
que le gouverneur de Terre-Neuve fasse voile le plus tôt possible... ${ }^{21}$.

Le 21 mars 1763, les Lords Commissaires du commerce et des plantations, suivant les instructions qu'ils avaient reçues, soumirent un avant-projet d'instructions à l'intention du gouverneur, comportant les modifications et les additions résultant des conditions du traité. Mais, dans leur lettre accompagnant cet avant-projet d'instructions, Charles Townshend et les autres membres du Conseil appelaient de nouveau l'attention sur le fait que

l'avant-projet d'instructions ne s'étend pas aux Iles du Cap Breton ou à St-Jean [I. P.-E.], ou à toutes parties des côtes de l'Acadie, du Canada ou du Labrador; car ces îles et cette étendue de territoire n'ayant jamais été inclues dans les limites des attributions du gouverneur de Terre-Neuve, ... nous n'avons pas cru qu'il nous était loisible d'étendre notre considération au-delà de nos attributions elles-mêmes ... .22.

Cette fois, lord Egremont fut impressionné par le danger que la côte du Labrador puisse ne faire l'objet d'aucune disposition visant à empêcher les Français de poursuivre leur ancien commerce avec les Indiens. Ni lui ni le Conseil du commerce ne semble avoir pensé à l'existence d'un mécanisme à Québec chargé du gouvernement du Labrador. Tout ce qui préoccupait le secrétaire d'État, c'était les réclamations de la Compagnie de la Baie d'Hudson.

D'après sa charte de 1670 , cette compagnie était propriétaire de "toutes les terres et de tous les territoires situés dans les contrées, sur les côtes et dans les confins des mers, des baies, des lacs, des rivières, des criques et des bras de mers se trouvant à l'embouchure du... détroit d'Hudson". Comme on l'a déjà indiqué, lorsqu'un groupe de marchands de Londres avait demandé qu'on leur concédât le littoral atlantique du Labrador

21 C.O. 194/15, f. 74. Egremont aux Lords du Commerce, Whitehall, 17 mars 1763.

22 C.O. 194/26, f. 77-8. Lords du Commerce à Egremont, Whitehall, 21 mars 1763. 
en 1752, cette requête avait été rejetée par le Conseil du commerce sous le prétexte qu'une ligne frontalière n'avait jamais été fixée entre les possessions britanniques et françaises dans ces régions. Cependant, la Compagnie de la Baie d'Hudson avait prétendu à l'époque que

le Labrador, dans toute son étendue... se trouve sur le détroit et la baie d'Hudson, et en constitue ainsi la côte,

et que, par conséquent, la compagnie avait "à juste titre le droit de réclamer... la dite étendue de terre appelée le Labrador ${ }^{23 "}$.

Le 23 mars 1763, Egremont demandait à la Compagnie de la Baie d'Hudson, par lettre, "quelles sont les limites sur la côte entre la compagnie de la Baie d'Hudson et la Côte du Labrador ?" Sir William Baker, gouverneur de la Compagnie, s'offrit aussitôt de "consulter" le secrétaire d'État en vue de discuter de cette affaire ${ }^{24}$. C'est par suite de cette discussion que lord Egremont semble avoir pris la décision de ne pas mentionner aucune "limite intérieure sur la côte", car immédiatement après, soit le 24 mars 1763, il retournait l'avantprojet d'instructions destiné au gouverneur de Terre-Neuve au Lord du Commerce, avec la note historique suivante:

Le Roi ayant jugé opportun que toute la côte du Labrador, de l'embouchure du détroit d'Hudson, jusqu'à la rivière St-Jean, qui débouche dans la mer, presque vis-à-vis l'extrémité occidentale de l'île d'Anticosti, y compris cette île, ainsi que toutes autres petites îles sur la dite côte du Labrador, ainsi que les Iles de la Madeleine dans le golfe du StLaurent, soient inclus dans le gouvernement de Terre-Neuve, j'ai reçu instruction de signifier à Vos Excellences le bon plaisir de Sa Majesté, à savoir que vous prépariez immédiatement, pour soumission au Roi afin qu'il y donne sa sanction royale, l'avant-projet d'une nouvelle Commission à l'intention du capitaine Thomas Graves, qui doit

23 Archives de la Compagnie de la Baie d'Hudson, Mémoire aux Lords du Commerce, 20 juilet 1752 (Joint App., VIII: 4098-9).

24 Archives de la Compagnie de la Baie d'Hudson, 23 mars 1763. (Joint App., VIII: 4110). 
être nommé gouverneur de l'Ile de Terre-Neuve et de la côte du Labrador ainsi que des diverses îles décrites ci-dessus ${ }^{25}$.

Le 29 mars 1763, Charles Townshend et le Conseil du commerce avaient un nouvel avant-projet de l'instruction à l'intention du gouverneur Graves. Les additions et les modifications de ce nouvel avant-projet révèlent le fond de leur pensée à l'égard du Labrador. Les Français ne devaient pas être autorisés à prendre et à sécher du poisson, du cap Bonavista jusqu'à Pointe Riche mais, à cette exception près, le gouverneur devait mettre "pleinement à exécution" la stipulation selon laquelle, dans le golfe du St-Laurent, ils ne devaient pas pêcher "sinon à une distance de trois lieues de toutes les côtes appartenant à la Grande-Bretagne, ainsi que de celles du continent et de celles des Îles". A cette même exception près, ils devaient "empêcher tous étrangers, quels qu'ils fussent, de pêcher ou de sécher du poisson sur l'une quelconque de ces côtes" relevant de son gouvernement. Il avait également instruction

d'ordonner à l'officier de tout navire relevant de votre commandement, qui pourrait être chargé de visiter cette partie de la côte du Labrador qui se trouve entre le détroit d'Hudson et le détroit de Belle Isle, d'inspecter et d'explorer la grande anse communément connue sous le nom d'Anse de Davis, afin de découvrir si elle ne renferme pas un passage vers la baie d'Hudson ou toute mer intérieure.

(Graves ne donna pas suite à cette dernière instruction, car aucun de ses navires n'était stationné sur cette côte. Bon nombre d'années devaient s'écouler avant que le gouvernement britannique se rendît compte que l'anse de Davis ne constituait qu'une des nombreuses baies qui caractérisent les côtes du Labrador).

Le gouverneur devait s'enquérir si un "établissement pourrait être nécessaire... en vue de... faire le commerce avec les Indiens... habitant la côte du Labrador".

Vous ne devez permettre aux sujets d'aucun prince ou Etat étranger de poursuivre quelque commerce

25 C.O. 194/15, Egremont aux Lords du Commerce, Whitehall, 24 $\operatorname{mars} 1763$. 
que ce soit avec lesdits Indiens, et de déployer tous vos efforts en vue de gagner leur affection, et de les induire à commercer avec nos sujets ...26".

La mise au point de ces instructions doit avoir été l'une des dernières activités de Charles Townshend pendant son bref séjour au Conseil du commerce. Le 8 avril 1763, lord Bute se retirait en tant que premier ministre. Il n'était entré au gouvernement qu'en vue de conclure la paix avec la France. C'était maintenant chose faite, et les adaptations administratives nécessaires résultant des conditions du traité étaient en voie de s'effectuer de façon satisfaisante. Son successeur comme premier ministre fut lord Grenville, beau-frère et ami intime de lord Egremont. L'obstiné et arrogant Townshend réclama plus d'influence au sein du nouveau gouvernement que Grenville n'était disposé à lui en concéder, de sorte qu'on lui donna à entendre que le Roi n'avait plus besoin de ses services. Il démissionna du Conseil du commerce et rejoignit Pitt dans l'Opposition, devenant un critique impitoyable de ses anciens collègues. Mais Egremont demeura l'un des secrétaires d'État, et la nouvelle Commission destinée au gouverneur Graves fut confirmée par le Grand Sceau de la Grande-Bretagne, tel que prévu, le 25 avril 1763. C'est donc à cette date que la côte du Labrador, pour la première fois, passait sous l'autorité du gouvernement de Terre-Neuve. Thomas Graves devenait ainsi "gouverneur et commandant en chef de notre dite Ile Terre-Neuve et de toutes les côtes du Labrador à partir de l'embouchure du détroit d'Hudson jusqu'à la rivière St-Jean ${ }^{27}$ ". L'homme responsable de cette décision était sir Charles Wyndham, deuxième comte d'Egremont qui, en sa qualité de secrétaire d'État, avait été responsable d'un traité de paix impopulaire, redonnant des libertés étendues aux Français, mais qui cherchait désormais à rendre applicables les conditions de ce traité. Sa décision visant à rattacher le Labrador à Terre-Neuve fut prise par suite de la pression exercée par les Lords Commissaires du commerce et des plantations, sous la présidence

26 C.O. 195/9, 164-296. Avant-projet d'instructions à l'intention de Graves, 29 mars 1763 (Joint App., II: 391-405).

27 Patent Roll, 3 Geo. III, Partie V, no 15. Commission 25 avril 1763 (Joint App., I: 149). 
de Charles Townshend, qui s'étaient opposés au traité de paix, et qui persistaient à vouloir empêcher les Français "de partager les avantages de ces pêcheries" ou "de leur ancien commerce avec les Indiens" du Labrador. Selon lui, la situation exigeait soit un établissement britannique quelconque dans ce territoire, soit un nombre suffisant de croiseurs pour saisir "tous les navires français s'approchant d'une certaine distance de cette côte". La seconde solution était plus facile à appliquer et semblait plus naturelle à un gouvernement dont le chef véritable était George III, ce roi dont l'attitude à l'égard des "établissements britanniques" devait plus tard entraîner la Révolution américaine.

\section{III. - PARTAGE DU LABRADOR EN 1763}

En prenant leur décision à propos du Labrador, rien n'indique qu'Egremont ou Townshend ait songé à la situation du gouverneur militaire du Québec. Ignorant tout de ce qui s'était passé, le général Murray continuait toujours d'encourager des aventuriers à s'adonner à la pêche au phoque. Le 26 avril 1763, le lendemain où la nouvelle commission fut délivrée à Graves, William Brymer, marchand de Québec, recevait "pour la pêche au phoque... cette étendue de terre sur la rive nord du StLaurent, communément appelée Cap Charles, en face de l'embouchure du détroit de Belle Isle", pour un minimum de quatre ans ${ }^{28}$, concession qui fut par la suite considérée tout à fait légale par le Conseil privé, malgré les efforts vigoureux du gouverneur Palliser, de Terre-Neuve et du Labrador, visant à la rendre nulle et non avenue ${ }^{29}$. Dans sa hâte à donner les nouvelles instructions au gouverneur Graves avant l'ouverture de la saison de la pêche, lord Egremont avait négligé de vérifier les pouvoirs déjà accordés (par l'entremise de l'Armée) au gouverneur Murray.

Le capitaine Thomas Graves, premier gouverneur de TerreNeuve, de la côte du Labrador et des Îles-de-la-Madeleine, avait

28 Rapport sur les postes ou terres concédés par le gouverneur Murray (Joint App., VII : 3671). III : 1046).

29 C.O. 195/15, 24. Pownall à Cooper, 16 novembre 1769 (Joint App., 
passé toute sa vie dans la Marine. Son père avait été contreamiral, et lui-même avait déjà accompli un assez long service dans la Marine, y compris deux ans en qualité de gouverneur de Terre-Neuve pendant la guerre. Au cours de l'été précédent, en 1762, il était entré en contact avec une force française à St-Jean, mais avec l'aide de renforts d'Halifax, il avait éventuellement eu la satisfaction de la mettre en fuite. Ce fut un meilleur gouverneur que la plupart de ceux qui furent envoyés à Terre-Neuve au cours de cette période. Sympathique envers les habitants de cette île, il favorisa, en fait, l'établissement d'un gouvernement civil, 70 ans environ avant que son avis soit finalement suivi à Londres. Par la suite, il joua un rôle éminent dans la guerre de la Révolution américaine et dans la guerre de la Révolution française. Devenu amiral en 1794, il fut élevé à la pairie lorsqu'il fut forcé de renoncer à son commandement par suite de blessures reçues en service actif contre les Français ${ }^{30}$.

Les Lords Commissaires de l'Amirauté donnèrent leurs instructions au gouverneur Graves, à Plymouth, en sa qualité de commandant en chef des navires de sa Majesté stationnés à Terre-Neuve, le 2 mai 1763. Au nombre des vaisseaux de guerre placés sous son commandement se trouvait le Pearl, qui avait l'ordre de passer la saison sur "la côte du Labrador, entre Belle Isle et la rivière St-Jean, et autour de l'île d'Anticosti", et le Lark, ("dans le détroit de Belle Isle et de là le long de la côte occidentale du Cap Ray"). C'était là les "croiseurs" que lè Conseil du commerce avait jugé nécessaires pour surveiller les Français. L'une des instructions données à Graves lui enjoignait

de déployer vos meilleurs efforts en vue d'encourager et d'appuyer la pêche à la baleine, dans le détroit de Belle Isle et plus particulièrement dans le port de York et dans les autres parties des côtes du Labrador, et de faire obstacle à tout commerce et à toute relation entre toutes personnes autre que les sujets de la Grande-Bretagne, avec les habitants de ce pays, qui, en droit, appartient uniquement à

30 Dictionary of National Biography, article sur Thomas Graves, baron Graves. 
Sa Majesté; et vous devrez également protéger la pêche au saumon et au phoque le long des côtes,... et régulariser et surveiller la pêche non seulement à Placentia et à St-Jean, mais aussi loin vers le Nord, le long des côtes de Terre-Neuve, et le long de celles du continent du Labrador, vu que les sujets de Sa Majesté s'y emploieront à pêcher et à sécher le poisson ${ }^{31}$.

Cette instruction révèle que l'extension de la pêche à partir des régions septentrionales de Terre-Neuve jusqu'à la côte du Labrador était présumée commencer immédiatement, si elle n'avait pas commencé déjà. Cet incident marqua l'inauguration d'une nouvelle période dans l'histoire de l'île ${ }^{32}$.

En août 1763, lord Egremont fut "foudroyé par une attaque d'apoplexie". Walpole fit observer à cette occasion que "chacun savait qu'il mourrait subitement; il ne prenait aucun exercice et on ne pouvait l'empêcher de manger". Cependant, la décision qu'il avait prise à propos du Labrador, et la frontière qu'il avait établie sur la côte, à l'intention du gouverneur de Terre-Neuve, furent acceptées par son successeur et annoncées publiquement dans une proclamation royale, qui fut publiée le 7 octobre 1763. Pour ce qui est du Québec, ses limites intérieures étaient nettement définies ainsi qu'il suit:

Le gouvernement du Québec, borné sur la côte du Labrador par la rivière St-Jean, et de là par une ligne tracée à partir de l'embouchure de cette rivière, et passant à travers le lac St-Jean, à l'extrémité sud du lac Nipissing; ...

Mais pour ce qui est de la "côte" qui avait été comprise dans le gouvernement de Terre-Neuve, la proclamation du 7 octobre, comme la commission de Graves en date du mois d'avril précédent, ne mentionnait nullement les limites intérieures:

Et afin que la pêche ouverte et libre de nos sujets puisse s'étendre et se poursuivre le long de la côte

31 Adm. Sec. Out Letters, Vol. 90 : 174-184 (Joint App., II: 406, 408).

32 Ralph Greenlee Lounsbury, The British Fishery at Newfoundland 1634-1763, (New Haven: Yale University Press, 1934), 320-321. 
du Labrador et autour des îles adjacentes, nous avons jugé oportun, de l'avis de notre dit Conseil privé, de placer toute cette côte, à partir de la rivière St-Jean jusqu'au détroit d'Hudson, ainsi que les îles d'Anticosti et de la Madeleine, et toutes les autres petites îles se trouvant le long de la dite côte, sous les soins et l'inspection de notre gouverneur de Terre-Neuve ${ }^{33}$.

Avec une logique douteuse, le comité judiciaire du Conseil privé en 1927 décida que le mot "côte" signifiait "ligne de partage des eaux"!

L'argument selon lequel on voulut que le territoire annexé à Terre-Neuve s'étendît jusqu'à la ligne de partage des eaux est confirmé par le fait que, dans la proclamation de 1763 , la province de Québec est décrite comme étant bornée au nord par une ligne tracée à partir de l'embouchure de la rivière St-Jean et se dirigeant vers l'ouest, - description qui porte à croire que le territoire à l'Est ou sur la rive gauche de la rivière St-Jean à partir de son embouchure jusqu'à la mer avait déjà été concédé au gouvernement de Terre-Neuve.

C'est ce qu'on peut lire dans le rapport du comité judiciaire du Conseil privé. Le fait plutôt étrange que la rivière St-Jean est une rivière relativement courte fut écarté de la façon suivante:

... la rivière St-Jean, ici mentionnée, ne s'élève pas en fait près de la ligne de partage des eaux mais à un certain point entre la hauteur de la terre et de la mer; mais il est clair, d'après les cartes de l'époque, que les sources de la rivière Romaine, qui s'élève à la ligne de partage des eaux et qui coule parallèlement à la rivière St-Jean, avaient été considérées comme étant les sources de cette dernière, et que la frontière orientale de la nouvelle province de Québec, à ce point-là, devait suivre le cours de la rivière Romaine, à partir de la ligne de partage des eaux jusqu'à la mer ${ }^{34}$.

33 (Joint App., I: 153-4).

34 In the Privy Council. In the Matter of ... Labrador, XII, Oral Proceedings : 1016. Report of the Judicial Committee. 
Le décret du conseil, daté du palais de Buckingham, le 22 mars 1927, mentionne par conséquent la rivière Romaine (et non la rivière St-Jean), et déclare que la partie de la frontière entre le Québec et Terre-Neuve est une ligne tracée

en direction nord le long de la rive gauche ou orientale de cette rivière et de son embouchure jusqu'à sa source et de là directement vers le Nord jusqu'à la crête de la ligne de partage des eaux ou à la hauteur des terres à cet endroit, et de là vers l'Ouest et vers le Nord le long de la crête de la ligne de partage des eaux et des rivières se jetant dans l'océan Atlantique jusqu'à ce qu'elles atteignent Cap Chidley ${ }^{35}$.

Lord Egremont, qui s'était abstenu délibérément de mentionner toutes "limites sur la côte entre la compagnie de la Baie d'Hudson et la côte du Labrador", aurait été étonné de cette décision. Il en eût été de même des auteurs de la proclamation de 1763 , qui n'avaient donné au public qu'une raison pour avoir placé "toute cette côte" sous l'autorité du gouverneur de Terre-Neuve: "que la pêche ouverte et libre de nos sujets puisse s'étendre et se poursuivre le long de la côte du Labrador"!

\section{IV. - CONCLUSION}

Les liens politiques entre Terre-Neuve et le Labrador commencèrent il $\mathrm{y}$ a exactement deux siècles. Le gouvernement britannique de l'époque cherchait simplement à conformer les instructions données au gouverneur de Terre-Neuve aux stipulations du traité de paix de 1763, qui avaient confirmé la victoire de la Grande-Bretagne sur la France. Lord Egremont avait agi avec hâte. Il s'agissait d'une décision anti-coloniale, vu qu'elle ne tenait nullement compte des intérêts des habitants ou propriétaires possibles établis sur la côte, et elle constituait en fait une décision visant à ce qu'aucun établissement ne s'y installe. L'extension de la "pêche ouverte et libre de nos sujets" au Labrador, en 1763, signifiait l'interdiction de toute propriété privée sur la côte et une tentative visant à ce qu'elle ne soit pas utilisée par des colons.

35 Ibid., XII : 1027. 
C'était également, il va sans dire, une décision anti-française. On tentait en effet d'assurer que le commerce français des fourrures au Labrador ne revive plus. Comme les pêcheurs de France étaient encore autorisés à sécher du poisson sur le Petit Nord, vis-à-vis le détroit de Belle-Isle, on estima nécessaire que le gouverneur de Terre-Neuve eût l'autorité de saisir tous navires français pouvant s'approcher de la côte du Labrador. Contrairement au gouverneur de Québec, le gouverneur de Terre-Neuve était un marin ayant des croiseurs à sa disposition. Tout comme la perte de la vallée du Mississipi, la perte de la côte du Labrador par le Québec fut l'un des résultats de la guerre de Sept Ans.

Cette décision de 1763 se révéla par la suite avantageuse pour les habitants de Terre-Neuve, vu qu'elle leur ouvrait un nouveau domaine de pêche. Ce n'est qu'en 1946, toutefois, que les habitants du Labrador furent considérés comme des citoyens de plein droit et qu'ils furent autorisés à voter, et ce fut alors par une décision directe du gouvernement britannique. En 1948, 78 p. 100 d'entre eux votaient en faveur de la Confédération avec le Canada. Depuis 1949, ils ont continué à manifester leur satisfaction générale en continuant à appuyer le gouvernement libéral de Terre-Neuve, que la Confédération mit sur pied.

Tant que la principale préoccupation des deux côtés du détroit de Belle-Isle demeurait la pêche, la décision de lord Egremont restait saine du point de vue géographique. Car pour les pêcheurs, ce n'est pas l'eau mais la terre qui constitue une barrière. Mais l'interprétation donnée au mot "côte" par le Conseil privé en 1927, suivie de la mise en valeur récente de vastes mines de fer à l'intérieur, a amené une nouvelle situation. $\mathrm{Du}$ point de vue politique, cette situation s'est manifestée en 1962 par la défaite inattendue du candidat du gouvernement dans le Labrador-Ouest. Le candidat victorieux avait, en 1959, contesté la circonscription de St-Jean-Sud, pour le compte du parti démocratique de Terre-Neuve, formé en vue de faire obstacle aux politiques ouvrières du gouvernement libéral de cette année-là. C'est là un indice que le mécontentement commence à se manifester dans cette nouvelle région minière. 
Entre-temps, faute de capitalistes privés importants, et pourtant fidèle aux principes de la propriété privée, qui était l'ordre naturel des choses à l'époque où régnait une petite économie fondée sur les efforts des pêcheurs individuels, Terre-Neuve permet à la grande majorité des bénéfices de la "côte du Labrador" d'aller à des capitalistes privés qui ne sont pas des TerreNeuviens ni, dans la plupart des cas, même des Canadiens. Il est vrai que cela rapporte à St-Jean des recettes de beaucoup supérieures à celles qu'on ait jamais pu réaliser au moyen des pêcheries. Terre-Neuve bénéficie actuellement beaucoup plus que jamais de la victoire de 1763 (et de 1927). Néanmoins, ces avantages économiques sont négligeables, si on les compare à ceux dont d'autres bénéficient par suite de l'exploitation des mines du Labrador, avantages qui échappent à la population du Canada dans son ensemble, y compris la population de TerreNeuve. Même du côté du Québec, le même problème se présente. Il s'agit là d'une situation qui démontre, de façon frappante, le danger qui peut désormais résulter de "l'autonomie provinciale" dans le domaine économique. Voilà l'un des problèmes de la Confédération. Les conditions ont changé radicalement depuis 1763 , alors que les seuls problèmes relatifs à la côte du Labrador consistaient à empêcher les Français de commercer avec les Indiens, et à étendre la pêche ouverte et libre aux sujets britanniques. Le grand problème du Canada, aujourd'hui, se rattache beaucoup plus à celui auquel Louis XIV faisait face en 1708; il s'agit, en somme, du problème qui consiste à maintenir l'existence indépendante de toute la région du golfe et du fleuve St-Laurent, et à en assurer l'extension vers l'Ouest en tant qu'unité politique et économique. Seul un gouvernement central pour toute la région, un gouvernement à Ottawa, pourrait disposer des ressources financières nécessaires à cette fin. L'autre solution consiste à accroître la subordination économique des dix provinces à l'égard des financiers étrangers. Comment peuton concilier ce fait à l'autre fait brutal selon lequel, à cause de l'expérience du passé, un si grand nombre de Canadiens craignent que leur culture locale distincte ne puisse survivre au sein d'un Canada économiquement uni? Ce régionalisme culturel pourrait encore moins survivre, semble-t-il, dans un Canada 
désuni. Il appartient à la majorité culturelle du pays de trancher ce dilemme, car elle seule est en mesure de supprimer toutes traces persistantes de la mentalité des vainqueurs de 1763.

GORDON O. ROTHNEY,

Doyen de la Faculté des Arts, Lakehead College of Arts, Science and Technology, Port Arthur, Ontario.

Traduit par Jacques Gouin, Chef adjoint,

Bureau des traductions,

Défense nationale, Ottawa. 